\title{
Synthesis Using Suzuki Cross Couplings of Sulfur Analogues of Dehydrotryptophan with a Definite Stereochemistry
}

\author{
Natália O. Silva, ${ }^{[a]}$ Ana S. Abreu, ${ }^{\text {[a] }}$ Paula M. T. Ferreira, ${ }^{\text {[a] }}$ Luís S. Monteiro, ${ }^{\text {[a] }}$ and \\ Maria-João R. P. Queiroz*[a]
}

Keywords: Amino acids / Sulfur heterocycles / Suzuki coupling / Stereoisomerism

Sulfur analogues of dehydrotryptophan (5-7) were prepared in moderate to good yields $(40-80 \%)$ by Suzuki cross coupling $\left[\mathrm{Pd}\left(\mathrm{PPh}_{3}\right)_{4}, \mathrm{Na}_{2} \mathrm{CO}_{3}\right.$ or $\left.\mathrm{NaHCO}_{3}, \mathrm{DME} / \mathrm{H}_{2} \mathrm{O}, 90{ }^{\circ} \mathrm{C}\right]$ of several benzo[b]thiophene boronic acids with the methyl esters of $N$-tert-butyloxycarbonyl- $\beta$-bromodehydroalanine [Boc- $\Delta \mathrm{Ala}(\beta-\mathrm{Br})-\mathrm{OMe}$ ] or $N$-tert-butyloxycarbonyl- $\beta$-bromodehydroaminobutyric acid [Boc- $\Delta \mathrm{Abu}(\beta-\mathrm{Br})-\mathrm{OMe}$ ]. The $\beta$ bromodehydroamino acid precursors 2 were, in turn, synthesized in high yields from the corresponding $N, N$-diacyldehydroamino acids 1 by treatment with trifluoroacetic acid (TFA) and $N$-bromosuccinimide (NBS) in two steps or in a one-pot procedure. Both procedures were stereoselective for the $E$ isomer of Boc- $\Delta \mathrm{Ala}(\beta-\mathrm{Br})-\mathrm{OMe}$. However, for Boc- $\Delta \mathrm{Abu}(\beta-$ $\mathrm{Br})-\mathrm{OMe}$, different ratios of $E / Z$ isomers were obtained in each procedure. The stereoselectivity for the Z-isomer was increased greatly with the one-pot procedure. Pure isomers were used in the coupling reactions and the stereochemistry of the starting material was generally maintained.

(c) Wiley-VCH Verlag GmbH, 69451 Weinheim, Germany, 2002)

\section{Introduction}

The insertion of non-proteinogenic conformationally constrained amino acids into bioactive peptides has proven to be a powerful tool in the elucidation of ligand-receptor binding interactions and in peptidomimetic design. ${ }^{[1]}$ Dehydroamino acids are among one of the most interesting classes of compounds that can be used for this purpose. These compounds are also found in several peptide antibiotics and are important substrates for the synthesis of novel amino acids through enantioselective catalytic hydrogenation. ${ }^{[2]}$ Although several methods have been described for their preparation, dehydroamino acids remain a challenging group of target molecules. Recently we have developed an efficient method for the stereoselective synthesis of the $Z$ isomers of $N, N$-diacyldehydroamino acid derivatives from the corresponding $\beta$-hydroxyamino acids. ${ }^{[3]}$ These compounds have proved to be versatile substrates in Michael addition reactions, allowing the preparation of several new $\beta$-substituted amino acids and also $\beta$-substituted dehydroamino acids. By this method it was possible to obtain several tryptophan and dehydrotryptophan analogues using $N$-unprotected indoles as nucleophiles. ${ }^{[4]}$

Here we report the use of our readily available $N, N$-diacyldehydroamino acid derivatives, after $N$-monodeprotection and $\beta$-bromination, in the Suzuki cross coupling reac-

\footnotetext{
[a] Departamento de Química-Universidade do Minho 4700-320 Braga Portugal

Fax: (internat.) +351-25/367-8983

E-mail: mjrpq@quimica.uminho.pt
}

tion with benzo[b]thiophene derivatives to obtain new sulfur analogues of dehydrotryptophan. This coupling reaction was chosen because it is one of the most powerful and versatile methods for $\mathrm{C}-\mathrm{C}$ bond formation as it: (i) tolerates a broad range of functionalities, (ii) can be performed under mild conditions, and (iii) usually maintains the stereochemistry of the starting material. ${ }^{[5]}$

\section{Results and Discussion}

The $Z$-isomers of the methyl ester of $N, N$-diacyldehydroamino acids 1 , obtained from the corresponding $\beta$-hydroxyamino acids, ${ }^{[3]}$ were $N$-monodeprotected and brominated in high yields to give $\beta$-bromo dehydroamino acids $\mathbf{2}$, using TFA and NBS either in two separate steps or in a one-pot procedure (Scheme 1). The latter gave slightly higher yields. In the case of Boc- $\Delta \mathrm{Ala}(\beta-\mathrm{Br})-\mathrm{OMe}$, using both methods, only the $E$-isomer was obtained. Other authors have reported the formation of only the $Z$-isomer when the amine function is monoprotected either with acety ${ }^{[6]}$ or with methyloxycarbonyl ${ }^{[7]}$ groups. Our result could be due to the steric hindrance produced by the bulky Boc group. Mixtures of $E / Z$ isomers were obtained in the synthesis of Boc$\Delta \mathrm{Abu}(\beta-\mathrm{Br})-\mathrm{OMe}$, with increased stereoselectivity for the $Z$-isomer, when the deprotection and bromination were carried out in a one-pot procedure (Scheme 1). This could result from the presence of TFA in the reaction mixture. The ratio of $E / Z$ isomers obtained using the two step procedure (1:1) is in agreement with the bromination performed by 
Hoerrner et al. ${ }^{[8]}$ on the methyl ester of $N$-acetyldehydroaminobutyric acid. The isomers were separated by column chromatography and the stereochemistry was determined using differential NOE enhancements between the $\beta$ ( $\Delta$ Ala) or $\gamma$ protons $(\triangle \mathrm{Abu})$ and the $\alpha \mathrm{NH}$.

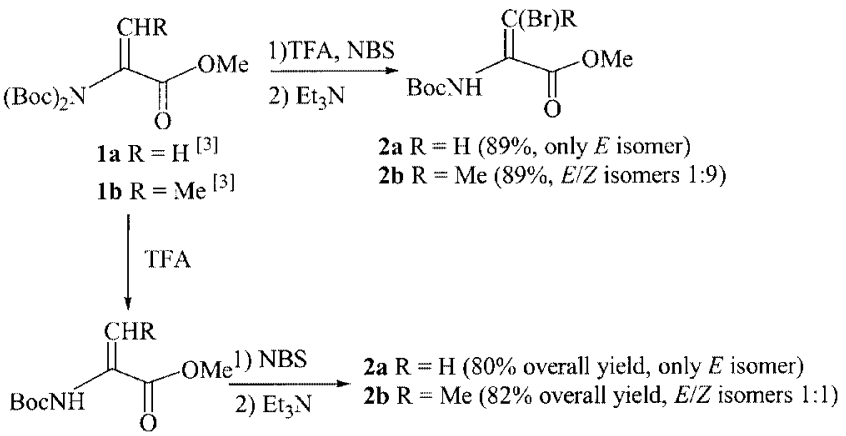

Scheme 1

The pure $\beta$-bromodehydroamino acids $(E)$-2a and $(E)$ - or (Z)-2b were prepared in order to be coupled under Suzuki coupling conditions with the commercially available benzo[ $b]$ thiophene 2- and 3-boronic acids and also with 7boronic-2,3-dimethylbenzo[b]thiophene acid (4). The latter was prepared in order to study the reactivity of the benzene ring of the benzo[b]thiophene system, to which less attention has been paid. Compound $\mathbf{4}$ was obtained from 7bromo-2,3-dimethylbenzo[b]thiophene (3) by halogen-lithium exchange, followed by boron transmetallation and treatment with acid (Scheme 2). Compound 3 was synthesized from 2-bromothiophenol and 3-bromobutan-2-one using $30 \% \mathrm{NaOH}$ in the first step and polyphosphoric acid (PPA) in the cyclodehydration step (Scheme 2). ${ }^{[9]}$

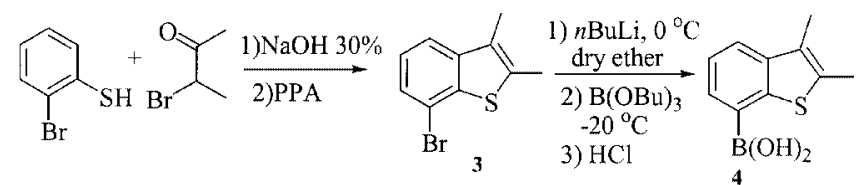

Scheme 2

The Suzuki coupling reactions were performed in DME/ water (4:1) using $\mathrm{NaHCO}_{3}$ (2 equiv.), or in DME and aqueous $2 \mathrm{M} \mathrm{Na}_{2} \mathrm{CO}_{3}$ (2 equiv.). ${ }^{[8]}$ In both cases $\mathrm{Pd}\left(\mathrm{PPh}_{3}\right)_{4}(10$ mol \%) was used as the palladium catalyst at $90{ }^{\circ} \mathrm{C}$ for between 4 and $7.5 \mathrm{~h}$ (Scheme 3, Table 1).

The coupled products were obtained in moderate to good yields (Table 1); the corresponding deboronated benzo[b]thiophenes were also isolated in small amounts (10-15\% yield). The results show a similar reactivity of both rings of the benzo[b]thiophene system and of both $\beta$-bromo dehydroamino acid derivatives.

The stereochemistry of Boc- $\Delta \mathrm{Ala}(\beta-\mathrm{Br})-\mathrm{OMe}$ and Boc$\Delta \mathrm{Abu}(\beta-\mathrm{Br})-\mathrm{OMe}$ was maintained, except in the case of the coupling of compound $\mathbf{4}$ with $(E)$-2b, in which $(E)-\mathbf{7 b}$ and

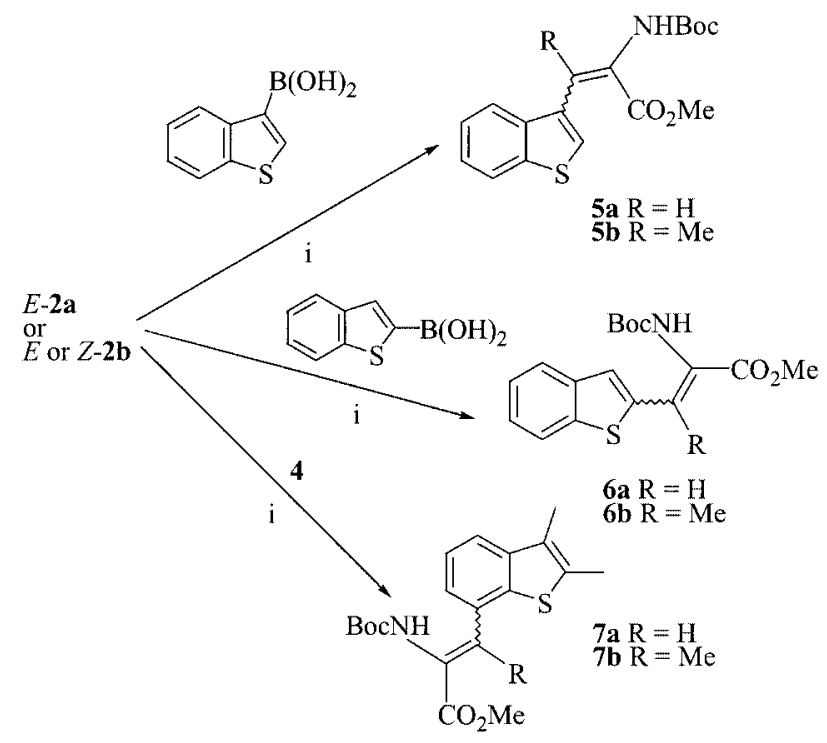

Scheme 3. i) $\mathrm{DME} / \mathrm{H}_{2} \mathrm{O}, \mathrm{Pd}\left(\mathrm{PPh}_{3}\right)_{4}(10 \mathrm{~mol} \%), \mathrm{Na}_{2} \mathrm{CO}_{3}$ or $\mathrm{NaHCO}_{3}$ (2 equiv.), $90{ }^{\circ} \mathrm{C}$

Table 1. Yields of the coupled products

\begin{tabular}{lllll}
\hline Benzo[b]thiophene & $\Delta$ aa & Base, time & Product & Yield (\%) \\
\hline 3-boronic acid & $(E)-\mathbf{2 a}$ & $\mathrm{Na}_{2} \mathrm{CO}_{3}, 4 \mathrm{~h}$ & $(E)-\mathbf{5 a}$ & 72 \\
& $(E)-\mathbf{2 b}$ & $\mathrm{Na}_{2} \mathrm{CO}_{3}, 7 \mathrm{~h}$ & $(E)-\mathbf{5 b}$ & 52 \\
& $(Z)-\mathbf{2 b}$ & $\mathrm{NaHCO}_{3}, 5 \mathrm{~h}$ & $(Z)-\mathbf{5 b}$ & 62 \\
\hline 2-boronic acid & $(E)-\mathbf{2 a}$ & $\mathrm{Na}_{2} \mathrm{CO}_{3}, 4 \mathrm{~h}$ & $(E)-\mathbf{6 a}$ & 81 \\
& $(E)-\mathbf{2 b}$ & $\mathrm{NaHCO}_{3}, 4 \mathrm{~h}$ & $(E)-\mathbf{6 b}$ & 61 \\
& $(Z)-\mathbf{2 b}$ & $\mathrm{NaHCO}_{3}, 6 \mathrm{~h}$ & $(Z)-\mathbf{6 b}$ & 47 \\
\hline 4 & $(E)-\mathbf{2 a}$ & $\mathrm{Na}_{2} \mathrm{CO}_{3}, 4 \mathrm{~h}$ & $(E)-\mathbf{7 a}$ & 40 \\
& $(E)-\mathbf{2 b}$ & $\mathrm{NaHCO}_{3}$, & $(E)-7 \mathbf{b}$ & 61 \\
& $7 \mathrm{~h} \mathrm{30} \mathrm{min}$ & $(Z)-7 \mathbf{b}$ & 7 \\
& & & \\
& $(Z)-\mathbf{2 b}$ & $\mathrm{NaHCO}_{3}$, & $(Z)-7 \mathbf{b}$ & 70 \\
& $5 \mathrm{~h} \mathrm{30} \mathrm{min}$ & &
\end{tabular}

$(Z)-7 \mathbf{b}$ were isolated in a ratio of 9:1. This could be due to base-catalysed isomerization ${ }^{[10]}$ to the thermodynamically more stable $Z$-isomer, since a longer reaction time was needed $(7.5 \mathrm{~h})$. With $(Z)-2 \mathbf{b}$ as starting material, and under the same reaction conditions, no isomerization occurred and $(Z)-7 \mathbf{b}$ was isolated in $70 \%$ yield after $5.5 \mathrm{~h}$ (Table 1$)$. In both cases 2,3-dimethyl-7-hydroxybenzo[b]thiophene ${ }^{[9]}$ was also isolated in $11 \%$ yield.

As in the case of the starting materials, the stereochemistry of the coupled products was determined by NOE experiments. For dehydroaminobutyric acid derivatives the proton chemical shifts (in $\mathrm{CDCl}_{3}$ ) of the methyl ester $(\mathrm{OMe})$ and of the $\gamma$ protons $(\mathrm{Me})$ of the $Z$-isomers always appear at higher values than those of the $E$-isomers (Table 2).

\section{Conclusion}

The Suzuki coupling reaction was applied successfully to the synthesis of both $E$ and $Z$ isomers of several sulfur ana- 
Table 2. ${ }^{1} \mathrm{H}$ NMR chemical shifts $\left(\mathrm{CDCl}_{3}\right)$ of the $\mathrm{OMe}$ and $\mathrm{Me}$ groups of coupled $\triangle \mathrm{Abu}$ derivatives

\begin{tabular}{lll}
\hline Compound & $\delta \mathrm{OMe}$ & $\delta \mathrm{Me}$ \\
\hline$(E)-\mathbf{5 b}$ & 3.34 & 2.19 \\
$(Z)-\mathbf{5 b}$ & 3.90 & 2.30 \\
$(E)-\mathbf{6 b}$ & 3.59 & 2.21 \\
$(Z)-\mathbf{6 b}$ & 3.87 & 2.45 \\
$(E)-\mathbf{7 b}$ & 3.37 & 2.21 \\
$(Z)-\mathbf{7 b}$ & 3.89 & 2.27 \\
\hline
\end{tabular}

logues of dehydrotryptophan. In this study the couplings were performed using benzo $[b]$ thiophenes functionalized either on the thiophene or on the benzene ring. In general the stereochemistry of the $\beta$-bromo dehydroamino acid derivatives was maintained.

Both stereoisomers of the coupled products obtained could be useful in structure-activity relationship studies aimed at elucidating the biologically active conformation of peptides and also in the development of new molecules of pharmaceutical interest.

\section{Experimental Section}

General Remarks: Melting points were determined on a Gallenkamp apparatus and are uncorrected. The ${ }^{1} \mathrm{H}$ NMR spectra were measured on a Varian Unity Plus spectrometer at $300 \mathrm{MHz}$. Spinspin decoupling techniques were used to assign the signals. The ${ }^{13} \mathrm{C}$ NMR spectra were measured on the same instrument at $75.4 \mathrm{MHz}$ (using DEPT $\theta 45^{\circ}$ ). Elemental analyses were determined on a LECO CHNS 932 elemental analyser.

Column chromatography was performed on Macherey-Nagel silica gel 230-400 mesh. Petroleum ether refers to the boiling range $40-60{ }^{\circ} \mathrm{C}$. When a solvent gradient was used the increase of polarity was done gradually from petroleum ether to mixtures of diethyl ether/petroleum ether increasing the amount of diethyl ether by $10 \%$ until the isolation of the product.

Preparation of the Methyl Esters of $N, N$-bis(tert-butyloxycarbonyl)dehydroamino Acids

Synthesis of $\mathrm{Boc}_{2}-\Delta \mathrm{Ala}-\mathrm{OMe}(1 \mathrm{a})$ and $\mathrm{Boc}_{2}-\Delta \mathrm{Abu}-\mathrm{OMe}(\mathbf{1 b})$ : These compounds were prepared from Boc-Ser-OMe and Boc-Thr-OMe, respectively, using the tert-butylpyrocarbonate/dimethylaminopyridine procedure described elsewhere. ${ }^{[3]}$

Monodeprotection of the Methyl Esters of $N, N$-bis(tert-butyloxycarbonyl)dehydroamino Acids

Synthesis of Boc- $\Delta$ Ala-OMe and Boc- $\Delta$ Abu-OMe: Compound 1a $(1.51 \mathrm{~g}, 5 \mathrm{mmol})$ or $\mathbf{1 b}(1.58 \mathrm{~g}, 5 \mathrm{mmol})$ was dissolved in dichloromethane $(0.1 \mathrm{M})$ and $2 \%$ of TFA was added slowly with vigorous stirring. The reaction was monitored by TLC and when no starting material was detected (ca. 1 hour) an additional $50 \mathrm{~mL}$ of dichloromethane was added. The organic phase was then washed with $\mathrm{NaHCO}_{3}(1 \mathrm{M})$ and brine $(3 \times 30 \mathrm{~mL}$ each $)$. After drying over $\mathrm{MgSO}_{4}$ the extract was taken to dryness at reduced pressure to afford Boc- $\Delta$ Ala-OMe $(0.98 \mathrm{~g}, 98 \%)$, as an oil, ${ }^{[3]}$ or Boc- $\Delta$ Abu-OMe $(0.96 \mathrm{~g}, 89 \%)$. Crystallization of the latter from diethyl ether $/ n$-hexane gave the pure $Z$-isomer as a white solid; m.p. $69.5-71.0^{\circ} \mathrm{C}$.
Bromination of the Methyl Esters of $\mathrm{N}$-tert-butyloxycarbonyldehydroamino Acids

Synthesis of Boc- $(\boldsymbol{E})-\Delta \mathrm{Ala}(\boldsymbol{\beta}-\mathrm{Br})-\mathrm{OMe}[(\boldsymbol{E})-\mathbf{2 a}]$ : Boc- $\Delta$ Ala-OMe $(1.01 \mathrm{~g}, 5 \mathrm{mmol})$ was dissolved in dichloromethane $(0.1 \mathrm{M})$ and 1.2 equiv. of $N$-bromosuccinimide were added with vigorous stirring. After reacting for 16 hours, triethylamine (1.5 equiv.) was added and stirring continued for an additional hour. The solvent was then evaporated at reduced pressure and the residue partitioned between $100 \mathrm{~mL}$ of dichloromethane and $50 \mathrm{~mL}$ of $\mathrm{KHSO}_{4}(1 \mathrm{M})$. The organic phase was washed with $\mathrm{KHSO}_{4}(1 \mathrm{M}), \mathrm{NaHCO}_{3}(1 \mathrm{M})$ and brine $\left(3 \times 30 \mathrm{~mL}\right.$ each). After drying over $\mathrm{MgSO}_{4}$ the extract was taken to dryness at reduced pressure to afford $(E)-2 \mathrm{a}(1.15 \mathrm{~g}, 82 \%)$ as a colourless oil. ${ }^{1} \mathrm{H} \mathrm{NMR}\left(\mathrm{CDCl}_{3}\right): \delta=1.47\left(\mathrm{~s}, 9 \mathrm{H}, \mathrm{CH}_{3} \mathrm{Boc}\right)$, $3.82\left(\mathrm{~s}, 3 \mathrm{H}, \mathrm{OCH}_{3}\right), 6.19$ (broad s, $\left.1 \mathrm{H}, \alpha \mathrm{NH}\right), 6.87(\mathrm{~s}, 1 \mathrm{H}, \beta \mathrm{CH})$ ppm. ${ }^{13} \mathrm{C} \mathrm{NMR}\left(\mathrm{CDCl}_{3}\right): \delta=27.78\left[\mathrm{C}\left(\mathrm{CH}_{3}\right)_{3}\right], 52.78\left(\mathrm{OCH}_{3}\right)$,

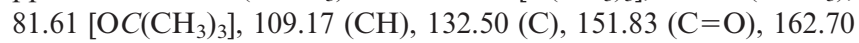
$(\mathrm{C}=\mathrm{O}) \mathrm{ppm}$.

Synthesis of Boc- $\Delta \mathrm{Abu}(\boldsymbol{\beta}-\mathrm{Br})-\mathrm{OMe}[(\boldsymbol{E})-\mathbf{2 b}$ and $(Z)-2 \mathrm{~b}]$ : The same procedure as described above was followed, substituting Boc- $\Delta$ AlaOMe for Boc- $\triangle \mathrm{Abu}-\mathrm{OMe}(1.08 \mathrm{~g}, 5 \mathrm{mmol})$ to give a $1: 1$ mixture of (E)-2b and (Z)-2b (1.35 g, 92\%). The diastereomers were separated by column chromatography using 30\% diethyl ether/petroleum ether as eluent.

(E)-2b: M.p. $108.0-109.0{ }^{\circ} \mathrm{C}$ (from diethyl ether $/ n$-hexane). ${ }^{1} \mathrm{H}$ $\operatorname{NMR}\left(\mathrm{CDCl}_{3}\right): \delta=1.46\left(\mathrm{~s}, 9 \mathrm{H}, \mathrm{CH}_{3} \mathrm{Boc}\right), 2.41\left(\mathrm{~s}, 3 \mathrm{H}, \gamma \mathrm{CH}_{3}\right)$, $3.82\left(\mathrm{~s}, 3 \mathrm{H}, \mathrm{OCH}_{3}\right), 6.00$ (broad s, $\left.1 \mathrm{H}, \alpha \mathrm{NH}\right) \mathrm{ppm} .{ }^{13} \mathrm{C} \mathrm{NMR}$ $\left(\mathrm{CDCl}_{3}\right): \delta=25.54\left(\mathrm{C}=\mathrm{CCH}_{3}\right), 28.02\left[\mathrm{C}\left(\mathrm{CH}_{3}\right)_{3}\right], 52.19\left(\mathrm{OCH}_{3}\right)$, $81.21\left[\mathrm{OC}\left(\mathrm{CH}_{3}\right)_{3}\right], 121.95(\mathrm{C}), 126.23(\mathrm{C}), 152.63(\mathrm{C}=\mathrm{O}), 164.45$ $(\mathrm{C}=\mathrm{O})$ ppm. $\mathrm{C}_{10} \mathrm{H}_{16} \mathrm{BrNO}_{4}$ (294.1): calcd. C 40.83, H 5.44, N 4.76; found C 40.92, H 5.43, N 4.88.

(Z)-2b: M.p. $75.5-77.0{ }^{\circ} \mathrm{C}$ (from $n$-hexane). ${ }^{1} \mathrm{H}$ NMR $\left(\mathrm{CDCl}_{3}\right)$ : $\delta=1.46\left(\mathrm{~s}, 9 \mathrm{H}, \mathrm{CH}_{3} \mathrm{Boc}\right), 2.52\left(\mathrm{~s}, 3 \mathrm{H}, \gamma \mathrm{CH}_{3}\right), 3.83(\mathrm{~s}, 3 \mathrm{H}$, $\left.\mathrm{OCH}_{3}\right), 6.23$ (broad s, $\left.1 \mathrm{H}, \alpha \mathrm{NH}\right) \mathrm{ppm} .{ }^{13} \mathrm{C} \mathrm{NMR}\left(\mathrm{CDCl}_{3}\right): \delta=$ $24.43 \quad\left(\mathrm{C}=\mathrm{CCH}_{3}\right), \quad 28.00 \quad\left[\mathrm{C}\left(\mathrm{CH}_{3}\right)_{3}\right], \quad 52.42 \quad\left(\mathrm{OCH}_{3}\right), \quad 81.46$ $\left[\mathrm{OC}\left(\mathrm{CH}_{3}\right)_{3}\right], 120.40(\mathrm{C}), 127.33(\mathrm{C}), 152.31(\mathrm{C}=\mathrm{O}), 163.17(\mathrm{C}=\mathrm{O})$ ppm. $\mathrm{C}_{10} \mathrm{H}_{16} \mathrm{BrNO}_{4}$ (294.1): calcd. C 40.83, H 5.44, N 4.76; found C 41.19, H 5.52, N 4.81 .

One-Pot Procedure for Synthesis of the $\beta$-Bromo $N$-tert-butyloxycarbonyldehydroamino Acid Methyl Esters

Synthesis of Boc- $(\boldsymbol{E})-\Delta \mathrm{Ala}(\boldsymbol{\beta}-\mathrm{Br})-\mathrm{OMe}[(\boldsymbol{E})-2 \mathrm{a}]: \mathrm{Boc}_{2}-\Delta \mathrm{Ala}-\mathrm{OMe}$ $(1.51 \mathrm{~g}, 5 \mathrm{mmol})$ was dissolved in dichloromethane $(0.1 \mathrm{M})$ and $2 \%$ of TFA was added slowly with vigorous stirring. The reaction was monitored by TLC and when no starting material was detected (ca. 1 hour) 1.2 equiv. of $N$-bromosuccinimide was added. After reacting for 16 hours triethylamine ( 3 equiv.) was added and stirring continued for an additional hour. The solvent was then evaporated at reduced pressure and the product $(E)$-2a $(1.25 \mathrm{~g}, 89 \%)$ was isolated as an oil, according to the procedure given above.

Synthesis of Boc- $\Delta \mathrm{Abu}(\boldsymbol{\beta}-\mathrm{Br})-\mathrm{OMe}[(\boldsymbol{E})-\mathbf{2 b}$ and $(Z)-\mathbf{2 b}]$ : The same procedure as described above was followed, substituting $\mathrm{Boc}_{2^{-}}$ $\Delta$ Ala-OMe for $\mathrm{Boc}_{2}-\Delta \mathrm{Abu}-\mathrm{OMe}(1.58 \mathrm{~g}, 5 \mathrm{mmol})$ to give $(E)-\mathbf{2 b}$ and $(Z)-2 \mathbf{b}(1.31 \mathrm{~g}, 89 \%)$ as a $1: 9$ mixture. Separation of the diastereomers was carried out by column chromatography using $30 \%$ diethyl ether/petroleum ether as eluent. Alternatively, (Z)-2b could be obtained by crystallization from diethyl ether $/ n$-hexane.

Synthesis of 7-Boronic-2,3-dimethylbenzo[b]thiophene Acid (4)

7-Bromo-2,3-dimethylbenzo[b]thiophene (3): A 30\% aqueous $\mathrm{NaOH}$ solution $(7 \mathrm{~mL}, 53 \mathrm{mmol})$ was added with stirring to 2-bromothiophenol (4.90 g, $26 \mathrm{mmol})$ at $0{ }^{\circ} \mathrm{C}$. After $30 \mathrm{~min}$ 3-bromobutan-2- 
one (3.70 g, $26 \mathrm{mmol}$ ) was added dropwise from an addition funnel and the mixture was left stirring for $30 \mathrm{~min}$ at room temp. Chloroform $(30 \mathrm{~mL})$ and water $(20 \mathrm{~mL})$ were then added and the phases separated. The aqueous phase was extracted with chloroform $(2 \times$ $30 \mathrm{~mL})$ and the combined organic phases were dried $\left(\mathrm{MgSO}_{4}\right)$, filtered and the solvent removed to give the corresponding ketoarylsulfide as a light yellow oil $(5.04 \mathrm{~g}, 75 \%) .{ }^{1} \mathrm{H} \mathrm{NMR}\left(\mathrm{CDCl}_{3}\right): \delta=$ $1.45\left(\mathrm{~d}, J=7.0 \mathrm{~Hz}, 3 \mathrm{H}, \mathrm{SCHCH}_{3}\right), 2.20\left(\mathrm{~s}, 3 \mathrm{H}, \mathrm{COCH}_{3}\right), 3.85$ (q, $\left.J=7.0 \mathrm{~Hz}, 1 \mathrm{H}, \mathrm{SCHCH}_{3}\right), 7.04($ broad t, $J=7.5 \mathrm{~Hz}, 1 \mathrm{H}, 4-$ H), 7.20 (broad t, $J=7.5 \mathrm{~Hz}, 1 \mathrm{H}, 5-\mathrm{H}), 7.32$ (broad d, $J=7.5 \mathrm{~Hz}$, $1 \mathrm{H}, 6-\mathrm{H}), 7.22$ (broad d, $J=7.5 \mathrm{H}, 1 \mathrm{~Hz}, 3-\mathrm{H})$. This compound $(5.00 \mathrm{~g}, 20 \mathrm{mmol})$ was added dropwise from an addition funnel to PPA (10 g) which had previously been heated at $150{ }^{\circ} \mathrm{C}$ for $15 \mathrm{~min}$. The mixture was then heated for $1 \mathrm{~h}$ at $150{ }^{\circ} \mathrm{C}$. After cooling, crushed ice was added $(30 \mathrm{~g})$ and it was left stirring until the ice had melted. Chloroform was added $(30 \mathrm{~mL})$ and the phases were separated. The aqueous phase was extracted with chloroform $(2 \times$ $50 \mathrm{~mL})$ and the organic phase was dried $\left(\mathrm{MgSO}_{4}\right)$, filtered and the solvent removed to give compound 3 (4.80 g, quantitative yield), m.p. $67.0-68.5{ }^{\circ} \mathrm{C}$ (from $n$-hexane). ${ }^{1} \mathrm{H}$ NMR $\left(\mathrm{CDCl}_{3}\right): \delta=2.29$ (s, $\left.3 \mathrm{H}, \mathrm{Ar}-\mathrm{CH}_{3}\right), 2.51\left(\mathrm{~s}, 3 \mathrm{H}, \mathrm{Ar}-\mathrm{CH}_{3}\right), 7.23$ (t, $J=8.0 \mathrm{~Hz}, 1 \mathrm{H}$, $5-\mathrm{H}), 7.42(\mathrm{dd}, J=8.0,1.0 \mathrm{~Hz}, 1 \mathrm{H}, 4-\mathrm{H}), 7.54(\mathrm{dd}, J=8.0,1.0 \mathrm{~Hz}$, $1 \mathrm{H}, 6-\mathrm{H}) \mathrm{ppm} .{ }^{13} \mathrm{C} \mathrm{NMR}\left(\mathrm{CDCl}_{3}\right): \delta=11.78\left(\mathrm{CH}_{3}\right), 13.77\left(\mathrm{CH}_{3}\right)$, $115.46(\mathrm{C}), 120.00(\mathrm{CH}), 125.16(\mathrm{CH}), 126.23(\mathrm{CH}), 132.88(\mathrm{C})$, 134.99 (C), 139.61 (C), 142.02 (C) ppm. $\mathrm{C}_{10} \mathrm{H}_{9} \mathrm{BrS}$ (241.1): calcd. C 49.81, H 3.76, S 13.29; found C 49.40, H 3.86, S 13.30 .

7-Boronic-2,3-dimethylbenzo[b]thiophene acid (4): A solution $1.6 \mathrm{M}$ of $n \mathrm{BuLi}$ in hexane $(5.1 \mathrm{~mL}, 8.1 \mathrm{mmol})$ was added dropwise to a solution of bromo compound $3(1.50 \mathrm{~g}, 6.2 \mathrm{mmol})$ in dry ether $(35 \mathrm{~mL})$ at $0{ }^{\circ} \mathrm{C}$, under argon. A white precipitate was formed and the mixture was left stirring for $20 \mathrm{~min}$ at $0{ }^{\circ} \mathrm{C}$. The temperature was lowered to $-20{ }^{\circ} \mathrm{C}$, tributyl borate was added $(2.2 \mathrm{~mL}$, $8.1 \mathrm{mmol}$ ), and the mixture was stirred at this temperature for 10 min, becoming a limpid solution that was left stirring for $2 \mathrm{~h}$ at room temp. The mixture was then cooled to $0{ }^{\circ} \mathrm{C}$ and acidified to $\mathrm{pH} 1-2$ with $1 \mathrm{~N} \mathrm{HCl}$. More ether was added and the mixture stirred for $15 \mathrm{~min}$. The phases were separated, and a $20 \%$ aqueous solution of $\mathrm{NaOH}(50 \mathrm{~mL})$ was added to the organic phase to form a white precipitate. The two phases were filtered under vacuum and a white solid was separated. This solid was completely dissolved with $1 \mathrm{~N} \mathrm{HCl}(20 \mathrm{~mL})$ and the mixture was extracted with ether (3 $\times 20 \mathrm{~mL}$ ). The combined organic phases were dried $\left(\mathrm{MgSO}_{4}\right)$, filtered and solvent removal gave compound 4 as a white solid $(0.60 \mathrm{~g}$, 47\%), m.p. $288.0-290.0^{\circ} \mathrm{C} .{ }^{1} \mathrm{H}$ NMR ([D 6$]$ DMSO): $\delta=2.24$ (s, 3 $\left.\mathrm{H}, \mathrm{Ar}-\mathrm{CH}_{3}\right), 2.42\left(\mathrm{~s}, 3 \mathrm{H}, \mathrm{Ar}-\mathrm{CH}_{3}\right), 7.31$ (apparent t, $J=7.0 \mathrm{~Hz}$, $1 \mathrm{H}, 5-\mathrm{H}), 7.65$ (broad d, $J=7.0 \mathrm{~Hz}, 1 \mathrm{H}, 4$ or $6-\mathrm{H}), 7.65$ (broad d, $J=7.0 \mathrm{~Hz}, 1 \mathrm{H}, 4$ or $6-\mathrm{H}), 8.30$ (broad s, $2 \mathrm{H}, 2 \times \mathrm{OH}) \mathrm{ppm}$. ${ }^{13} \mathrm{C}$ NMR ([D $]$ DMSO): $\delta=11.07\left(\mathrm{CH}_{3}\right), 13.33\left(\mathrm{CH}_{3}\right), 109.30(\mathrm{C})$, $123.04(\mathrm{CH}), 123.14(\mathrm{CH}), 125.61(\mathrm{C}), 130.15(\mathrm{CH}), 134.33(\mathrm{C})$, 140.23 (C), 143.16 (C) ppm.

General Procedure for Palladium-Catalysed Cross Couplings of Benzo[b]thiophene Boronic Acids with $\beta$-Bromo Dehydroamino Acid Derivatives: Compounds $(E)-\mathbf{2 a},(E)-\mathbf{2 b}$ or $(Z)-\mathbf{2 b}(1 \mathrm{mmol})$ were coupled with benzo $[b]$ thiophene boronic acids (1.1 equiv.) in a mixture of either DME/water $(4 \mathrm{~mL} / 1 \mathrm{~mL})$ and $\mathrm{NaHCO}_{3}$ (2 equiv.) or DME $(5 \mathrm{~mL})$ and aqueous $2 \mathrm{M} \mathrm{Na}_{2} \mathrm{CO}_{3}$ (2 equiv.), and $\mathrm{Pd}\left(\mathrm{PPh}_{3}\right)_{4}$ (10 mol \%). The reactions were followed by TLC which determined the different reaction times (Table 1). After cooling, diethyl ether and water were added and the phases were separated. The aqueous phase was washed with diethyl ether $(3 \times 50 \mathrm{~mL})$. The combined organic phases were dried $\left(\mathrm{MgSO}_{4}\right)$, filtered and solvent removal gave a brown residue which was submitted to column chromato- graphy affording the coupled products. The corresponding deboronated benzo $[b]$ thiophenes were isolated as the less polar products in $10-15 \%$ yield.

Boc- $(E)-\Delta \mathrm{Ala}[\boldsymbol{\beta}-($ benzo[b]thiophen-3-yl)]-OMe $\quad[(E)-5 a)]$ : Column chromatography using a solvent gradient from neat petroleum ether to $40 \%$ diethyl ether/petroleum ether gave compound $(E)-5$ a as a white solid $(0.24 \mathrm{~g}, 72 \%)$, m.p. $158.0-159.0{ }^{\circ} \mathrm{C} .{ }^{1} \mathrm{H}$ NMR $\left(\mathrm{CDCl}_{3}\right)$ : $\delta=1.41\left(\mathrm{~s}, 9 \mathrm{H}, \mathrm{CH}_{3} \mathrm{Boc}\right), 3.91\left(\mathrm{~s}, 3 \mathrm{H}, \mathrm{OCH}_{3}\right), 6.17(\mathrm{~s}, 1 \mathrm{H}, \alpha-$ NH), 7.40-7.47 (m, 2 H, $2 \times$ Ar-H), 7.58 (s, 1 H, 2-H), 7.86-7.92 $(\mathrm{m}, 3 \mathrm{H}, 2 \times \mathrm{Ar}-\mathrm{H}$ and $\beta-\mathrm{CH}) \mathrm{ppm} .{ }^{13} \mathrm{C} \mathrm{NMR}\left(\mathrm{CDCl}_{3}\right): \delta=28.07$ $\left[\mathrm{C}\left(\mathrm{CH}_{3}\right)_{3}\right], 52.65\left(\mathrm{OCH}_{3}\right), 81.14\left[\mathrm{OC}\left(\mathrm{CH}_{3}\right)_{3}\right], 121.61(\mathrm{CH}), 121.74$ $(\mathrm{CH}), 122.72(\mathrm{CH}), 124.65(\mathrm{CH}), 124.92(\mathrm{CH}), 125.31(\mathrm{C}), 128.28$ $(\mathrm{CH}), 129.19$ (C), 138.24 (C), 139.20 (C), 153.21 (C=O), 165.91 $(\mathrm{C}=\mathrm{O})$ ppm. $\mathrm{C}_{17} \mathrm{H}_{19} \mathrm{NO}_{4} \mathrm{~S}$ (333.4): calcd. $\mathrm{C} 61.24, \mathrm{H}$ 5.74, N 4.20, $\mathrm{S} 9.62$; found C 61.12, H 5.77, N 4.23, S 9.52.

Boc- $(E)-\Delta$ Ala $[\beta-(b e n z o[b]$ thiophen-2-yl)]-OMe $\quad[(E)-6 a]$ : Column chromatography using a solvent gradient from neat petroleum ether to $40 \%$ diethyl ether/petroleum ether gave compound $(E)-6 \mathbf{a}$ as a white solid $(0.27 \mathrm{~g}, 81 \%)$, m.p. $150.0-151.0{ }^{\circ} \mathrm{C} .{ }^{1} \mathrm{H}$ NMR $\left(\mathrm{CDCl}_{3}\right)$ : $\delta=1.50\left(\mathrm{~s}, 9 \mathrm{H}, \mathrm{CH}_{3} \mathrm{Boc}\right), 3.87\left(\mathrm{~s}, 3 \mathrm{H}, \mathrm{OCH}_{3}\right), 5.98(\mathrm{~s}, 1 \mathrm{H}, \alpha-$ NH), 7.25-7.44 (m, 2 H, $2 \times$ Ar-H), $7.55(\mathrm{~s}, 1 \mathrm{H}, 3-\mathrm{H}), 7.74$ (broad s, $1 \mathrm{H}, \beta-\mathrm{CH}), 7.77-7.86(\mathrm{~m}, 2 \mathrm{H}, 2 \times \mathrm{Ar}-\mathrm{H}) \mathrm{ppm} .{ }^{13} \mathrm{C} \mathrm{NMR}$ $\left(\mathrm{CDCl}_{3}\right): \delta=28.20\left[\mathrm{C}\left(\mathrm{CH}_{3}\right)_{3}\right], 52.53\left(\mathrm{OCH}_{3}\right), 81.25\left[\mathrm{OC}\left(\mathrm{CH}_{3}\right)_{3}\right]$, $122.21(\mathrm{CH}), 124.28(\mathrm{CH}), 124.69(\mathrm{CH}), 125.91(\mathrm{CH}), 128.52(\mathrm{CH})$, $129.73(\mathrm{CH}), 134.99(\mathrm{C}), 136.67(\mathrm{C}), 138.29(\mathrm{C}), 141.83(\mathrm{C}), 153.60$ $(\mathrm{C}=\mathrm{O}), 165.51(\mathrm{C}=\mathrm{O}) \mathrm{ppm} . \mathrm{C}_{17} \mathrm{H}_{19} \mathrm{NO}_{4} \mathrm{~S}$ (333.4): calcd. $\mathrm{C} 61.24$, H 5.74, N 4.20, S 9.62; found C 61.00, H 5.74, N 4.07, S 9.31.

Boc- $(E)-\Delta$ Ala[ $[\beta-(2,3-d i m e t h y l b e n z o[b]$ thiophen-7-yl)]-OMe $[(E)-7 \mathrm{a}]$ : Column chromatography using a solvent gradient from neat petroleum ether to $40 \%$ diethyl ether/petroleum ether gave the product as a white solid $(0.14 \mathrm{~g}, 40 \%)$, m.p. $125.0-126.0{ }^{\circ} \mathrm{C} .{ }^{1} \mathrm{H}$ NMR $\left(\mathrm{CDCl}_{3}\right): \delta=1.34$ (s, $\left.9 \mathrm{H}, \mathrm{CH}_{3} \mathrm{Boc}\right), 2.31$ (s, $\left.3 \mathrm{H}, \mathrm{Ar}-\mathrm{CH}_{3}\right), 2.50$ (s, $\left.3 \mathrm{H}, \mathrm{Ar}-\mathrm{CH}_{3}\right), 3.90\left(\mathrm{~s}, 3 \mathrm{H}, \mathrm{OCH}_{3}\right), 6.25(\mathrm{~s}, 1 \mathrm{H}, \alpha-\mathrm{NH})$, 7.33-7.39 (m, $2 \mathrm{H}, \beta-\mathrm{CH}$ and 5-H), 7.50-7.60 (m, $2 \mathrm{H}$, 4- and 6H) ppm. ${ }^{13} \mathrm{C} \mathrm{NMR}\left(\mathrm{CDCl}_{3}\right): \delta=11.45\left(\mathrm{CH}_{3}\right), 13.74\left(\mathrm{CH}_{3}\right), 27.99$ $\left[\mathrm{C}\left(\mathrm{CH}_{3}\right)_{3}\right], 52.67\left(\mathrm{OCH}_{3}\right), 80.99\left[\mathrm{OC}\left(\mathrm{CH}_{3}\right)_{3}\right], 121.71(\mathrm{CH}), 123.47$ $(\mathrm{CH}), 124.00(\mathrm{CH}), 125.28(\mathrm{CH}), 126.23(\mathrm{C}), 127.43(\mathrm{C}), 128.19$ (C), 133.79 (C), 138.47 (C), 141.49 (C), 152.45 (C=O), 165.77 (C= O) ppm. $\mathrm{C}_{19} \mathrm{H}_{23} \mathrm{NO}_{4} \mathrm{~S}(361.5)$ : calcd. C 63.14, H 6.41, N 3.88, S 8.87; found C 63.39, H 6.57, N 3.88, S 8.68.

Boc- $(Z)-\Delta$ Abu[ $\beta-($ benzo[b]thiophen-2-yl)]-OMe $\quad[(E)-6 b]$ : Column chromatography using a solvent gradient from neat petroleum ether to $30 \%$ diethyl ether/petroleum ether gave product $(E)-6 \mathbf{b}(0.21 \mathrm{~g}$, $61 \%$ ). Crystallization from diethyl ether $/ n$-hexane gave white crystals, m.p. $116.0-118.0{ }^{\circ} \mathrm{C} .{ }^{1} \mathrm{H}$ NMR $\left(\mathrm{CDCl}_{3}\right): \delta=1.49(\mathrm{~s}, 9 \mathrm{H}$, $\left.\mathrm{CH}_{3} \mathrm{Boc}\right), 2.21\left(\mathrm{~s}, 3 \mathrm{H}, \gamma-\mathrm{CH}_{3}\right), 3.59\left(\mathrm{~s}, 3 \mathrm{H}, \mathrm{OCH}_{3}\right), 6.14(\mathrm{~s}, 1 \mathrm{H}$, $\alpha-\mathrm{NH}), 7.14$ (s, $1 \mathrm{H}, 3-\mathrm{H}), 7.30-7.41(\mathrm{~m}, 2 \mathrm{H}, 2 \times \mathrm{Ar}-\mathrm{H})$, $7.68-7.82(\mathrm{~m}, 2 \mathrm{H}, 2 \times \mathrm{Ar}-\mathrm{H}) \mathrm{ppm} .{ }^{13} \mathrm{C} \mathrm{NMR}\left(\mathrm{CDCl}_{3}\right): \delta=21.00$

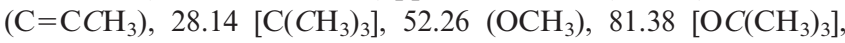
$122.07(\mathrm{CH}), 122.53(\mathrm{CH}), 123.54(\mathrm{CH}), 124.35(\mathrm{CH}), 124.43(\mathrm{CH})$, 125.50 (C), 126.55 (C), 139.46 (C), 139.96 (C), 142.62 (C), 152.67 $(\mathrm{C}=\mathrm{O}), 165.95(\mathrm{C}=\mathrm{O}) \mathrm{ppm} . \mathrm{C}_{18} \mathrm{H}_{21} \mathrm{NO}_{4} \mathrm{~S}$ (347.4): calcd. $\mathrm{C} 62.23$, H 6.09, N 4.03, S 9.23; found C 62.29, H 6.10, N 4.00, S 8.99.

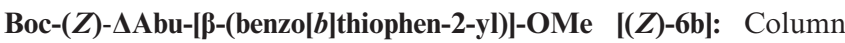
chromatography using a solvent gradient from neat petroleum ether to $40 \%$ diethyl ether/petroleum ether gave $(Z)-6 \mathbf{b}$ as a white solid $(0.16 \mathrm{~g}, 47 \%)$. Crystallization from diethyl ether $/ n$-hexane gave white crystals m.p.108.0-110.0 ${ }^{\circ} \mathrm{C} .{ }^{1} \mathrm{H}$ NMR $\left(\mathrm{CDCl}_{3}\right): \delta=1.48(\mathrm{~s}$, $9 \mathrm{H}, \mathrm{CH}_{3} \mathrm{Boc}$ ), 2.45 (s, $\left.3 \mathrm{H}, \gamma-\mathrm{CH}_{3}\right), 3.87\left(\mathrm{~s}, 3 \mathrm{H}, \mathrm{OCH}_{3}\right), 6.20$ (s, $1 \mathrm{H}, \alpha-\mathrm{NH}), 7.32-7.42(\mathrm{~m}, 2 \mathrm{H}, 2 \times \mathrm{Ar}-\mathrm{H}), 7.49(\mathrm{~s}, 1 \mathrm{H}, 3-\mathrm{H})$, 7.76-7.86 (m, $2 \mathrm{H}, 2 \times \mathrm{Ar}-\mathrm{H}) \mathrm{ppm} .{ }^{13} \mathrm{C} \mathrm{NMR}\left(\mathrm{CDCl}_{3}\right): \delta=19.37$ 
$\left(\mathrm{C}=\mathrm{CCH}_{3}\right), 28.19\left[\mathrm{C}\left(\mathrm{CH}_{3}\right)_{3}\right], 52.21\left(\mathrm{OCH}_{3}\right), 81.21\left[\mathrm{OC}\left(\mathrm{CH}_{3}\right)_{3}\right]$, $121.97(\mathrm{CH}), 123.94(\mathrm{CH}), 124.57(\mathrm{CH}), 125.28(\mathrm{CH}), 129.06(\mathrm{C})$, 138.79 (C), 140.53 (C), 140.98 (C), $153.38(\mathrm{C}=\mathrm{O}), 165.90(\mathrm{C}=\mathrm{O})$ ppm. $\mathrm{C}_{18} \mathrm{H}_{21} \mathrm{NO}_{4} \mathrm{~S}$ (347.4): calcd. C 62.23, H 6.09, N 4.03, S 9.23; found C 62.29, H 6.10, N 4.00, S 8.99.

Boc- $(E)-\Delta \mathrm{Abu}[\boldsymbol{\beta}$-(benzo[b]thiophen-3-yl)]-OMe $\quad[(E)-5 \mathrm{~b}]:$ Column chromatography using a solvent gradient from neat petroleum ether to $20 \%$ diethyl ether/petroleum ether gave the product as a white solid $\left(0.18\right.$ g, 52\%), m.p. $120.0-122.0{ }^{\circ} \mathrm{C} .{ }^{1} \mathrm{H} \mathrm{NMR}\left(\mathrm{CDCl}_{3}\right): \delta=$ $1.51\left(\mathrm{~s}, 9 \mathrm{H}, \mathrm{CH}_{3} \mathrm{Boc}\right), 2.19\left(\mathrm{~s}, 3 \mathrm{H}, \gamma-\mathrm{CH}_{3}\right), 3.34\left(\mathrm{~s}, 3 \mathrm{H}, \mathrm{OCH}_{3}\right)$, $6.17(\mathrm{~s}, 1 \mathrm{H}, \alpha-\mathrm{NH}), 7.19(\mathrm{~s}, 1 \mathrm{H}, 2-\mathrm{H}), 7.30-7.40(\mathrm{~m}, 2 \mathrm{H}, 2 \times$ Ar-H), 7.62-7.70 (m, $1 \mathrm{H}$, Ar-H) 7.82-7.88 (m, $1 \mathrm{H}$, Ar-H) ppm. ${ }^{13} \mathrm{C} \mathrm{NMR}\left(\mathrm{CDCl}_{3}\right): \delta=20.93\left(\mathrm{C}=\mathrm{CCH}_{3}\right), 28.19\left[\mathrm{C}\left(\mathrm{CH}_{3}\right)_{3}\right], 51.84$ $\left(\mathrm{OCH}_{3}\right), 80.95\left[\mathrm{OC}\left(\mathrm{CH}_{3}\right)_{3}\right], 122.45(\mathrm{CH}), 122.64(\mathrm{CH}), 124.19$ $(\mathrm{CH}), 124.31(\mathrm{CH}), 126.23(\mathrm{C}), 132.40(\mathrm{C}), 136.50(\mathrm{C}), 137.81(\mathrm{C})$, $139.67(\mathrm{C}), 153.06(\mathrm{C}=\mathrm{O}), 165.44(\mathrm{C}=\mathrm{O})$ ppm. $\mathrm{C}_{18} \mathrm{H}_{21} \mathrm{NO}_{4} \mathrm{~S}$ (347.4): calcd. C 62.23, H 6.09, N 4.03, S 9.23; found C 62.34, H 6.19, N 4.07, S 9.17.

Boc-( $Z$ )- $\Delta$ Abu[ $\beta$-(benzo[b]thiophen-3-yl)]-OMe $[(Z)-5 b]$ : Column chromatography using a solvent gradient from neat petroleum ether to $30 \%$ diethyl ether/petroleum ether gave the product as a white solid $(0.22$ g, $62 \%)$ m.p. $122.0-124.0{ }^{\circ} \mathrm{C} .{ }^{1} \mathrm{H}$ NMR $\left(\mathrm{CDCl}_{3}\right): \delta=$ 1.39 (s, $\left.9 \mathrm{H}, \mathrm{CH}_{3} \mathrm{Boc}\right), 2.30\left(\mathrm{~s}, 3 \mathrm{H}, \gamma-\mathrm{CH}_{3}\right), 3.90\left(\mathrm{~s}, 3 \mathrm{H}, \mathrm{OCH}_{3}\right)$, $5.65(\mathrm{~s}, 1 \mathrm{H}, \alpha-\mathrm{NH}), 7.32-7.43(\mathrm{~m}, 3 \mathrm{H}, 3 \times \mathrm{Ar}-\mathrm{H}), 7.58-7.65(\mathrm{~m}$, $1 \mathrm{H}, \mathrm{Ar}-\mathrm{H}) 7.86-7.93(\mathrm{~m}, 1 \mathrm{H}, \mathrm{Ar}-\mathrm{H}) \mathrm{ppm} .{ }^{13} \mathrm{C} \mathrm{NMR}\left(\mathrm{CDCl}_{3}\right)$ : $\delta=20.74\left(\mathrm{C}=\mathrm{CCH}_{3}\right), 27.99\left[\mathrm{C}\left(\mathrm{CH}_{3}\right)_{3}\right], 52.03\left(\mathrm{OCH}_{3}\right), 80.67$ $\left[\mathrm{OC}\left(\mathrm{CH}_{3}\right)_{3}\right], 122.74(\mathrm{CH}), 124.50(\mathrm{CH}), 124.72(\mathrm{CH}), 125.85(\mathrm{C})$, 127.87 (C), 135.37 (C), 136.17 (C), 140.03 (C), 153.02 (C=O), $165.45(\mathrm{C}=\mathrm{O})$ ppm. $\mathrm{C}_{18} \mathrm{H}_{21} \mathrm{NO}_{4} \mathrm{~S}$ (347.4): calcd. $\mathrm{C}$ 62.23, H 6.09, N 4.03, S 9.23; found C 62.05, H 6.21, N 4.00, S 9.08.

Boc-(Z)- $\Delta$ Abu[ $[\beta-(2,3-d i m e t h y l b e n z o[b]$ thiophen-7-yl)]-OMe $\quad[(Z)-$ 7b]: Column chromatography using a solvent gradient from neat petroleum ether to $30 \%$ diethyl ether/petroleum ether gave the product as a white solid $(0.26 \mathrm{~g}, 70 \%)$, m.p. $116.0-118.0{ }^{\circ} \mathrm{C} .{ }^{1} \mathrm{H}$ NMR $\left(\mathrm{CDCl}_{3}\right): \delta=1.35$ (s, $9 \mathrm{H}, \mathrm{CH}_{3} \mathrm{Boc}$ ), 2.27 (s, $\left.3 \mathrm{H}, \gamma-\mathrm{CH}_{3}\right)$, $2.32\left(\mathrm{~s}, 3 \mathrm{H}, \mathrm{Ar}-\mathrm{CH}_{3}\right) 2.47\left(\mathrm{~s}, 3 \mathrm{H}, \mathrm{Ar}-\mathrm{CH}_{3}\right), 3.89\left(\mathrm{~s}, 3 \mathrm{H}, \mathrm{OCH}_{3}\right)$, $5.70(\mathrm{~s}, 1 \mathrm{H}, \alpha-\mathrm{NH}), 7.10$ (broad signal, $1 \mathrm{H}, 6-\mathrm{H}$ ), 7.39 (apparent $\mathrm{t}, J=7.6 \mathrm{~Hz}, 1 \mathrm{H}, 5-\mathrm{H}) 7.56($ broad d, $J=7.9 \mathrm{~Hz}, 1 \mathrm{H}, 4-\mathrm{H})$ ppm. ${ }^{13} \mathrm{C} \mathrm{NMR}\left(\mathrm{CDCl}_{3}\right): \delta=11.49\left(\mathrm{CH}_{3}\right), 13.71\left(\mathrm{CH}_{3}\right), 19.63(\mathrm{C}=$ $\left.\mathrm{CCH}_{3}\right), 28.04\left[\mathrm{C}\left(\mathrm{CH}_{3}\right)_{3}\right], 52.08\left(\mathrm{OCH}_{3}\right), 80.60\left[\mathrm{OC}\left(\mathrm{CH}_{3}\right)_{3}\right], 120.74$ $(\mathrm{CH}), 122.53(\mathrm{CH}), 124.52(\mathrm{CH}), 125.24(\mathrm{C}), 127.49(\mathrm{C}), 131.41$ (C), 133.62 (C), 134.35 (C), 135.82 (C), 141.57 (C), 153.03 (C=O), $165.56(\mathrm{C}=\mathrm{O})$ ppm. $\mathrm{C}_{20} \mathrm{H}_{25} \mathrm{NO}_{4} \mathrm{~S}$ (375.5): calcd. C 63.98, H 6.71, N 3.73, S 8.54; found C 63.65, H 6.78, N 3.84, S 8.44.

7-Hydroxy-2,3-dimethylbenzo[b]thiophene ${ }^{[9]}(0.02 \mathrm{~g}, 11 \%)$ was also isolated, eluting from the column after the deboronated compound and before the coupled product.
Boc- $(E)-\Delta$ Abu-[ $\beta-(2,3-d i m e t h y l b e n z o[b]$ thiophen-7-yl)]-OMe $\quad[(E)-$ 7b]: Column chromatography using a solvent gradient from neat petroleum ether to $30 \%$ diethyl ether/petroleum ether gave $(Z)-7 \mathbf{b}$ as a white solid $(0.03 \mathrm{~g}, 7 \%)$, followed by $(E)-7 \mathbf{b}$, also as a white solid $(0.23 \mathrm{~g}, 61 \%)$ m.p. $116.0-118.0{ }^{\circ} \mathrm{C} .{ }^{1} \mathrm{H}$ NMR $\left(\mathrm{CDCl}_{3}\right)$ : $\delta=$ 1.51 (s, $\left.9 \mathrm{H}, \mathrm{CH}_{3} \mathrm{Boc}\right), 2.21$ (s, $\left.3 \mathrm{H}, \gamma-\mathrm{CH}_{3}\right), 2.29$ (s, $\left.3 \mathrm{H}, \mathrm{Ar}-\mathrm{CH}_{3}\right)$, 2.46 (s, $\left.3 \mathrm{H}, \mathrm{Ar}-\mathrm{CH}_{3}\right), 3.37$ (s, $\left.3 \mathrm{H}, \mathrm{OCH}_{3}\right), 6.12$ (s, $\left.1 \mathrm{H}, \alpha-\mathrm{NH}\right)$, 7.03 (broad d, $J=7.3 \mathrm{~Hz}, 1 \mathrm{H}, 6-\mathrm{H}$ ), 7.33 (apparent t, $J=7.9$, $7.3 \mathrm{~Hz}, 1 \mathrm{H}, 5-\mathrm{H}), 7.50(\mathrm{dd}, J=7.9,0.9 \mathrm{~Hz}, 1 \mathrm{H}, 4-\mathrm{H}) \mathrm{ppm} .{ }^{13} \mathrm{C}$ NMR $\left(\mathrm{CDCl}_{3}\right): \delta=11.48\left(\mathrm{CH}_{3}\right), 13.71\left(\mathrm{CH}_{3}\right), 20.50\left(\mathrm{C}=\mathrm{CCH}_{3}\right)$, $28.21\left[\mathrm{C}\left(\mathrm{CH}_{3}\right)_{3}\right], 51.77\left(\mathrm{OCH}_{3}\right), 80.79\left[\mathrm{OC}\left(\mathrm{CH}_{3}\right)_{3}\right], 120.10(\mathrm{CH})$, $122.01(\mathrm{CH}), 123.93(\mathrm{CH}), 125.12(\mathrm{C}), 127.22(\mathrm{C}), 133.66(\mathrm{C})$, 135.51 (C), 136.15 (C), 139.01 (C), 141.12 (C), 153.09 (C=O), $164.95(\mathrm{C}=\mathrm{O})$ ppm. $\mathrm{C}_{20} \mathrm{H}_{25} \mathrm{NO}_{4} \mathrm{~S}$ (375.5): calcd. $\mathrm{C}$ 63.98, H 6.71, N 3.73, S 8.54; found C 63.87, H 6.79, N 3.76, S 8.47.

7-Hydroxy-2,3-dimethylbenzo[b]thiophene ${ }^{[9]}(0.02 \mathrm{~g}, 11 \%)$ was also isolated, eluting from the column after the deboronated compound and before the coupled products.

\section{Acknowledgments}

The Foundation for Science and Technology (Portugal) is thanked for financial support through IBQF-Univ. Minho, POCTI/99/QUI/ 32689 project (also financial support of N.O. Silva) and through SFRH/BD/4709/2001 (PhD financial support of A.S. Abreu).

[1] [1a] A. Giannis, T. Kolten, Angew. Chem. 1993, 105, 1303-1326; Angew. Chem. Int. Ed. Engl. 1993, 32, 1244-1267. ${ }^{[1 \mathrm{~b}]}$ M. P. Paradisi, I. Torrini, G. P. Zecchini, G. Lucente, Z. Gavuzzo, F. Mazza, G. Pochetti, Tetrahedron 1995, 51, 2379-2386. ${ }^{[1 \mathrm{c}]}$ S. E. Gibson, N. Guille, M. J. Tozer, Tetrahedron 1999, 55, 585-615.

[2] A. Hammadi, H. Lam, M. Gondry, A. Ménez, R. Genet, Tetrahedron 2000, 56, 4473-4477.

[3] P. M. T. Ferreira, H. Maia, L. S. Monteiro, J. Sacramento, J. Chem. Soc., Perkin Trans. 1 1999, 3697-3703.

[4] [4a] P. M. T. Ferreira, H. Maia, L. S. Monteiro, J. Sacramento, J. Sebastião, J. Chem. Soc., Perkin Trans. 1 2000, 3317-3324. [4b] P. M. T. Ferreira, H. Maia, L. S. Monteiro, J. Sacramento, J. Chem. Soc., Perkin Trans. 1 2001, 3167-3172.

[5] [5a] A. Suzuki, N. Miyaura, Chem. Rev. 1995, 95, 2457-2483. ${ }^{[5 b]}$ A. Suzuki, J. Organomet. Chem. 1999, 576, 147-168.

[6] M. J. Burk, J. G. Allen, W. F. Kiesman, K. M. Stoffan, Tetrahedron Lett. 1997, 38, 1309-1312.

[7] B. Miossec, R. Danion-Bougot, D. Daniou, Synthesis 1994, $1171-1174$.

${ }^{[8]}$ R. Scott Hoerrner, D. Askin, R. P. Volante, P. J. Reider, Tetrahedron Lett. 1998, 39, 3455-3458.

[9] M.-J. R. P. Queiroz, R. Dubest, J. Aubard, R. Faure, R. Guglielmetti, Dyes and Pigments 2000, 47, 219-229.

${ }^{[10]}$ V. Calò, A. Nacci, A. Monopoli, L. Lopez, A. di Cosmo, Tetrahedron 2001, 57, 6071-6077.

Received March 6, 2002

[O02119] 